environment subject to the conditions of the Creative Commons Attribution-NonCommercial-ShareAlike

licence < http://creativecommons.org/licenses/by-nc-sa/2.5/>. The written permission of

Cambridge University Press must be obtained for commercial re-use.

\title{
Outreach and screening following the 2005 London bombings: usage and outcomes
}

\author{
C. R. Brewin ${ }^{1 *}$, N. Fuchkan ${ }^{1}$, Z. Huntley ${ }^{1}$, M. Robertson ${ }^{2}$, M. Thompson ${ }^{2}$, P. Scragg ${ }^{2}$, P. d'Ardenne ${ }^{3}$ \\ and A. Ehlers ${ }^{4}$ \\ ${ }^{1}$ Clinical, Educational and Health Psychology, University College London, UK \\ ${ }^{2}$ Traumatic Stress Clinic, Camden and Islington NHS Foundation Trust, London, UK \\ ${ }^{3}$ Institute of Psychotrauma, East London NHS Foundation Trust, London, UK \\ ${ }^{4}$ Centre for Anxiety Disorders and Trauma, Institute of Psychiatry, London, UK
}

Background. Little is known about how to remedy the unmet mental health needs associated with major terrorist attacks, or what outcomes are achievable with evidence-based treatment. This article reports the usage, diagnoses and outcomes associated with the 2-year Trauma Response Programme (TRP) for those affected by the 2005 London bombings.

Method. Following a systematic and coordinated programme of outreach, the contact details of 910 people were obtained by the TRP. Of these, 596 completed a screening instrument that included the Trauma Screening Questionnaire (TSQ) and items assessing other negative responses. Those scoring $\geqslant 6$ on the TSQ, or endorsing other negative responses, received a detailed clinical assessment. Individuals judged to need treatment $(n=217)$ received trauma-focused cognitive-behaviour therapy (TF-CBT) or eye movement desensitization and reprocessing (EMDR). Symptom levels were assessed pre- and post-treatment with validated self-report measures of post-traumatic stress disorder (PTSD) and depression, and 66 were followed up at 1 year.

Results. Case finding relied primarily on outreach rather than standard referral pathways such as primary care. The effect sizes achieved for treatment of DSM-IV PTSD exceeded those usually found in randomized controlled trials (RCTs) and gains were well maintained an average of 1 year later.

Conclusions. Outreach with screening, linked to the provision of evidence-based treatment, seems to be a viable method of identifying and meeting mental health needs following a terrorist attack. Given the failure of normal care pathways, it is a potentially important approach that merits further evaluation.

Received 5 October 2009; Revised 15 January 2010; Accepted 18 January 2010; First published online 9 March 2010

Key words: CBT, outreach, PTSD, screening, terrorism.

\section{Introduction}

Although it is well established that, following terrorist attacks, a minority of individuals develop a persistent mental health problem, most commonly posttraumatic stress disorder (PTSD; Call \& Pfefferbaum, 1999; Whalley \& Brewin, 2007; Neria et al. 2008; WTC Medical Working Group, 2008), the evidence base for planning an effective response is lacking. In the UK it has been assumed that those requiring specialist treatment will be catered for by referrals from general practitioners (GPs) to existing services, but the effectiveness or otherwise of this care pathway has never been investigated. Following the 1995 Oklahoma City

* Address for correspondence: Dr C. R. Brewin, Clinical, Educational and Health Psychology, University College London, Gower Street, London WC1E 6BT, UK.

(Email : c.brewin@ucl.ac.uk) bombing, direct victims with more serious disorders are reported to have been under-served in terms of screening, triage, referral to specialist services for established treatments, and subsequent monitoring (Pfefferbaum et al. 2002). Similarly, the latest report of the World Trade Center Registry documents high rates of continuing mental health needs 7 years after the attacks of 11 September 2001 (WTC Medical Working Group, 2008). To overcome this failure to meet treatment needs, early and proactive outreach has been recommended (Turner et al. 1989; Brewin, 2001), but never formally implemented. We report on the activity and outcomes associated with a clinically led programme set up immediately following the 2005 London bombings to test the feasibility of implementing outreach and screening linked to evidence-based treatment.

The London bombings consisted of a series of linked incidents that stretched over 17 days from 7 to 
23 July 2005, including both successful and failed attempts to detonate bombs and the shooting of an innocent passenger on an underground train. The 7 July attack was the largest mass casualty event in the UK since World War Two, resulting in 775 casualties and 52 deaths, and there were substantial psychological effects on the population of London (Rubin et al. 2005). Seven months later much of this distress was reduced, but a minority reported persistent stress reactions and behavioural changes, with $61 \%$ reporting that they saw the world differently as a result of the bombings (Rubin et al. 2007). The public mental health strategy, adopted in London for the first time, was to assume that elevated symptoms in the majority of the local population would not be long-lasting, to systematically locate and screen individuals directly involved in the bombings, and to offer specialist, National Institute for Clinical Excellence (NICE)recommended interventions to those who were not recovering naturally. The Trauma Response Programme (TRP), funded by the Department of Health, ran from September 2005 to September 2007. It consisted of two elements: a dedicated four-person screening and assessment team and additional clinical psychology sessions in specialist post-traumatic stress centres. The 2-year programme was run by a multi-professional steering group and was responsible to a project board that monitored and approved all costs incurred.

To assess the need for outreach we report whether referrals for treatment came through the expected route (i.e. GPs), on the take-up of screening, and on the prevalence of disorders requiring treatment. Only one previous study has investigated the outcome of evidence-based treatment initiated within 3 months of a terrorist attack (Gillespie et al. 2002) and it is not known whether these gains are maintained over time. We therefore investigated the clinical outcomes achieved by the TRP by collecting pre-treatment, posttreatment and 1-year follow-up data, and comparing our outcomes with the results of published randomized controlled trials (RCTs) for PTSD.

\section{Method}

\section{Design}

The programme design has been described previously (Brewin et al. 2008). This was a single-cohort study, as the ethical, financial and organizational constraints of mounting an immediate and unplanned-for postdisaster response, and also the desire to overcome barriers to treatment, precluded the use of control groups or of enrolling individuals in a research trial. Ethical approval was received from Barnet, Enfield and Haringey Local Research Ethics Committee
(LREC) and informed consent was obtained for nonaudit data.

\section{Screening}

Contact details of individuals known to have been involved in the bombings were available from a wide variety of sources including NHS Direct, hospitals, charitable relief funds, and the police. All GPs in the greater London area received two letters from the Department of Health alerting them to the likelihood of mental health problems following exposure to the London bombings, and to the existence of the programme. A media campaign advertised the programme and encouraged individuals to contact it. All individuals identified received a letter or telephone call and a brief two-page questionnaire containing screening questions to detect any current symptoms of psychopathology. Screening for PTSD used the Trauma Screening Questionnaire (TSQ; Brewin et al. 2002), which enquires about the presence of 10 PTSD symptoms in the past week. Responses to each item are either 'Yes, at least twice' or 'No'. Previous research has demonstrated that it has excellent performance relative to other PTSD screening instruments and that endorsement of $\geqslant 6$ symptoms yields high levels of sensitivity and specificity (Brewin, 2005; Walters et al. 2007). Other screening questions used a similar 'Yes/No' format and included two validated depression items (Kroenke et al. 2003) and three specially constructed questions asking about travel phobia and about increased smoking and drinking following exposure to the bombings. A final question asked whether respondents had had any other response to the bombings that concerned them.

\section{Clinical assessment}

Individuals screening positive on the TSQ or endorsing any of the additional screening items were invited to attend for a more detailed assessment that included the SCID-IP (First et al. 1997). This longer clinical assessment determined suitability for trauma-focused treatment in terms of meeting criteria for a DSM-IV or ICD-10 disorder that was related to being exposed to the bombings and that was not resolving of its own accord. Conditions not meeting full diagnostic criteria were also eligible if they were persistent and were associated with significant distress or impairment. Based on the self-reported trajectory of symptoms, a clinical decision was made whether to refer for immediate treatment or to continue monitoring in the expectation that recovery would occur naturally. In the latter case, individuals were screened again at 3-, 6- and 9-monthly intervals. 


\section{Treatment}

Consistent with recently published guidelines (NICE, 2005), two treatments for PTSD were used: traumafocused cognitive-behaviour therapy (TF-CBT) and eye movement desensitization and reprocessing (EMDR). All lead clinicians of the participating treatment centres met monthly to ensure that treatments were provided with uniform quality and in strict adherence with these guidelines. Rather than working from specific treatment manuals, clinicians were required to implement the individual TF-CBT or EMDR protocols used in their specialist centre and received ongoing supervision from experienced trauma clinicians there. The most commonly used approach was TF-CBT ( $>80 \%$ of patients treated). A minority of patients received either a combination of TF-CBT and EMDR $(\sim 10 \%)$ or EMDR only $(<10 \%)$. People with other disorders received evidence-based interventions for that disorder, mainly TF-CBT. There were no restrictions on the number of sessions. Patients in each of the three centres completed the symptom items from the Post-traumatic Diagnostic Scale (PDS; Foa et al. 1997) and the Beck Depression Inventory (BDI; Beck et al. 1979) before treatment started and at subsequent sessions until treatment was finished. There are 17 items scored $0-3$ on the PDS, yielding a possible range of $0-51$, and 21 items scored $0-3$ on the BDI, yielding a possible range of $0-63$. Scores from the final session that a patient attended were used as posttreatment measures.

\section{Follow-up}

As part of a separate evaluation attempts were made to follow up as many individuals who had been referred to treatment within the programme as possible. Within the lifetime of the project, 101 were followed up. This subsample did not differ from the 248 originally referred for treatment in age, gender, ethnicity or symptom scores pre- and post-treatment $(p>0.05)$. Of these 101 participants, 10 never entered treatment and 10 never completed treatment according to their therapist. Follow-up outcome measures were collected on 66 out of 81 individuals who completed treatment, the median time being 390 days after treatment ended (range 48-793 days).

\section{Statistical analyses}

Changes in the PDS and BDI were tested with ANOVA and $t$ tests separately for the intentionto-treat and completer samples. There were two measures of effect size. In addition to Cohen's $d$ (the difference between the pre-treatment and posttreatment means divided by their common standard deviation), we report an alternative, more conservative measure, which is the mean pre-post difference divided by the standard deviation of the mean difference. Reliable change (improvement beyond that expected by chance) and clinically significant change (reliable change, with patients additionally moving from being closer to the mean of a dysfunctional population at pre-treatment to being closer to the mean of a functional population at post-treatment) were calculated using the formulae of Jacobson \& Truax (1991). Thresholds for the PDS were calculated from original validation data provided in Foa et al. (1997), yielding for reliable change a fall of at least 8.04 and a cut-off between populations of 23.36. Corresponding thresholds for the BDI (reliable change 8.46, cut-off point 14.29) were taken from Seggar et al. (2002). As the dysfunctional samples of Foa et al. and Seggar et al. had a mean PDS and BDI score that best matched our subsample with DSM-IV PTSD, indices were only applied to this group.

\section{Results \\ Contact with the screening and assessment team}

Figure 1 provides a flow diagram that illustrates how individuals involved in the bombings whose identity became known to the programme progressed through screening, assessment and treatment. The programme obtained contact information and sent screening materials to 910 adults, mostly within the first 6 months. Only $4.3 \%$ of these contacts were as a result of GP referrals. Contact information came mainly from lists of survivors held by NHS hospitals and clinics $(41.7 \%)$, from police witness lists $(12.0 \%)$, from other organizations involved in the response to the bombings such as the 7 July Assistance Centre and the Red Cross $(27.1 \%)$, and from occupational health departments $(4.8 \%)$. In addition, individuals self-referred $(5.8 \%)$ or referred relatives and friends $(3.3 \%)$ as a result of seeing information in the mass media.

Of this sample, $65.5 \%$ returned at least one screening questionnaire. The average age of the screened sample was 41.6 years (S.D. $=12.2$ years), and $45 \%$ were male. Although most had been involved in the bombings on 7 July, some had been present at the other key events in the period stretching up until 23 July. According to the questionnaire, $32.8 \%$ said they had been injured, $57.9 \%$ believed that they might have been injured or killed, $16.9 \%$ said that a family member or close friend was injured or killed, and $73.8 \%$ claimed to have personally witnessed the effect of one of the bombings.

Of the 596 who returned a screening questionnaire, $56.7 \%$ screened positive at the first or subsequent 


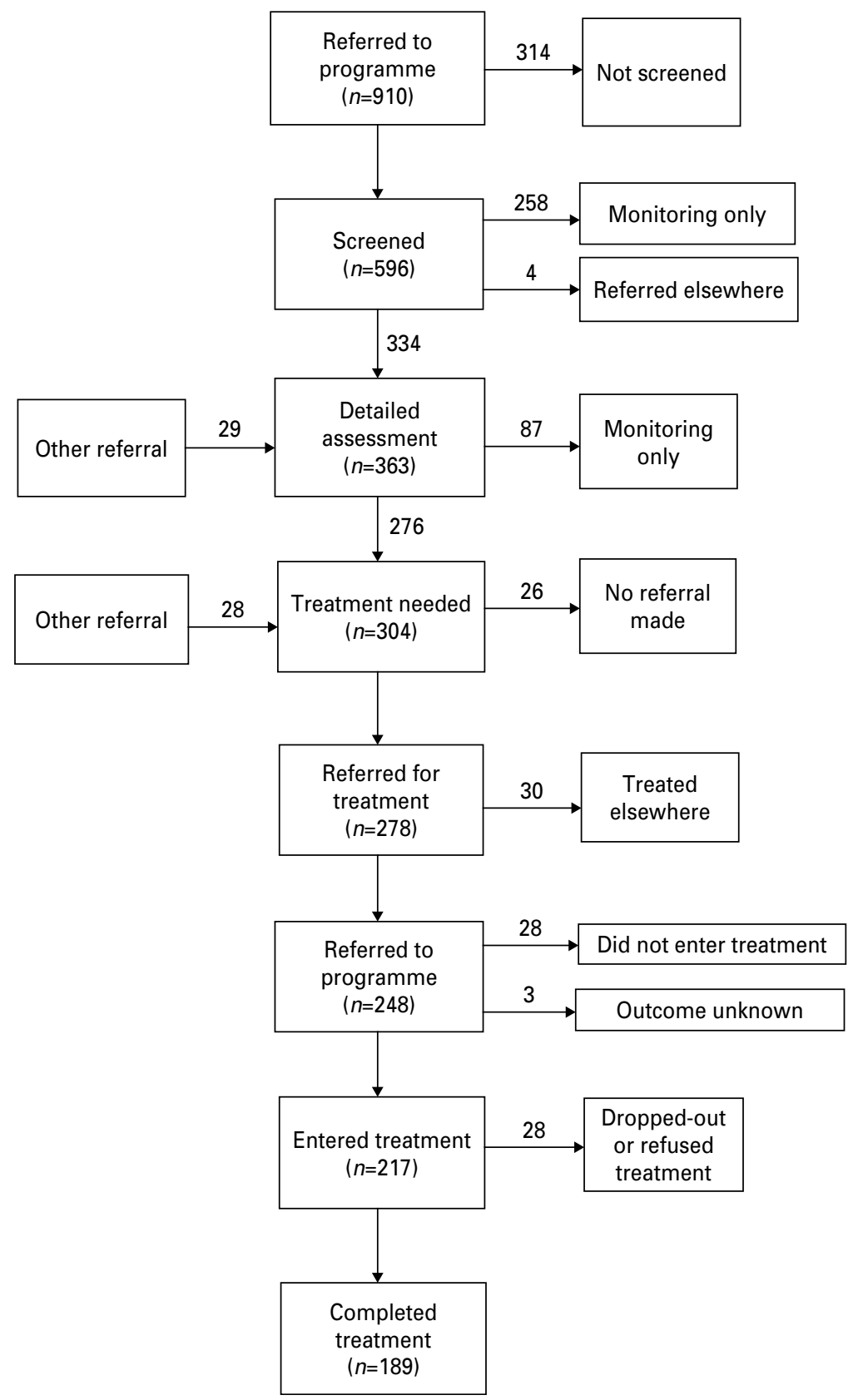

Fig. 1. Flow of participants through screening and treatment.

screening and received a detailed clinical assessment. The most common primary diagnosis was DSM-IV PTSD, often co-morbid with other disorders $(n=149$; $41 \%)$. In addition, a further $40(11 \%)$ met criteria for ICD-10 PTSD but not DSM-IV PTSD (all cases of DSMIV PTSD met criteria for ICD-10 PTSD). Other diagnoses included specific (travel) phobia $(n=28 ; 7.7 \%)$, depression without PTSD $(n=17 ; 4.7 \%)$, adjustment disorder $(n=15 ; 4.1 \%)$, complicated grief $(n=11$; $3.0 \%)$ and generalized anxiety disorder $(n=6 ; 1.7 \%)$. Data on the existence of previous mental disorder were available on 304 of those assessed and indicated that $16.1 \%$ had had a disorder.

In total, 304 people were known to the programme to be in need of treatment for psychological problems related to the London bombings. Being judged in need of treatment was unrelated to reporting a previous mental disorder $\left[\chi^{2}(1, n=304)=1.61\right.$, N.s.]. Twenty-six were not referred, mainly because they did not want treatment at that time. Of the 278 people who were referred for psychological therapy, 30 were treated nearer to where they lived and were not assessed 
Table 1. Treatment outcome details for all diagnoses

\begin{tabular}{|c|c|c|c|c|c|c|}
\hline & $n$ & $\begin{array}{l}\text { Pre-treatment, } \\
\text { mean (s.D.) }\end{array}$ & $\begin{array}{l}\text { Post-treatment, } \\
\text { mean (S.D.) }\end{array}$ & $\begin{array}{l}\text { Effect size } \\
\text { (Cohen's } d \text { ) }\end{array}$ & $\begin{array}{l}\text { Effect size based } \\
\text { on difference score } \\
\text { (s.D. of difference) }\end{array}$ & $\begin{array}{l}\text { Follow-up, } \\
\text { mean }(n, \text { S.D. })\end{array}$ \\
\hline \multicolumn{7}{|c|}{ Intention-to-treat sample } \\
\hline PDS & 208 & $29.1(11.2)$ & $11.3(11.2)$ & 1.59 & $1.48(12.0)$ & - \\
\hline BDI & 208 & $21.7(11.3)$ & $9.9(10.3)$ & 1.09 & $1.12(10.6)$ & - \\
\hline \multicolumn{7}{|c|}{ Completer sample } \\
\hline PDS & 181 & $28.9(11.4)$ & $9.6(10.3)$ & 1.77 & $1.68(11.5)$ & $11.8(62,11.4)$ \\
\hline BDI & 182 & $21.3(11.5)$ & $8.5(9.4)$ & 1.22 & $1.28(10.1)$ & $9.8(64,9.9)$ \\
\hline
\end{tabular}

PDS, Post-traumatic Diagnostic Scale; BDI, Beck Depression Inventory; S.D., standard deviation.

further. A total of 248 were referred to the three participating specialist post-traumatic stress centres. Of these 248, contact was lost prior to treatment with 31 patients, leaving 217 patients who entered treatment. Of these, 101 were men and 116 were women. Their average age was 40.6 years (range 22-71, s.D. $=$ 10.1 years). Self-identified ethnicity was White British, White Irish or White Other (69.7\%), Black African, Black Caribbean or Black Other $(4.6 \%)$, Asian Indian or Chinese $(4.2 \%)$, and Other $(8.4 \%) ; 13.4 \%$ did not indicate their ethnic background.

\section{Treatment}

The sample as a whole attended an average of 11.9 sessions (range 1-59, S.D. =9.9) and missed 1.9 sessions (range 0-15, S.D.=2.6). Twenty-eight (13\%) patients dropped out or refused treatment after entering the treatment programme, leaving a sample of 189 who completed treatment. Reporting previous mental health disorder was unrelated to the number of sessions attended, or to change achieved on the PDS or BDI [largest $t(175-203)=1.35$, N.s.]. To enable comparisons to be made with the research literature, outcome data are presented separately for the sample as a whole (all diagnoses) and for those specifically meeting DSM-IV criteria for PTSD.

\section{All diagnoses}

Table 1 shows the scores on the PDS and BDI separately for the entire (intention-to-treat) sample, including those who dropped out, and the (completer) sample who received a full course of treatment. Intention-to-treat data were imputed from the last observation carried forward (LOCF), and an ANOVA on the PDS scores showed statistically highly significant improvement $[F(1,207)=455.8, p<0.001]$. There was similar improvement on the BDI scores
$[F(1,207)=261.8, p<0.001]$. Table 1 also shows the substantial effect size for these treatment gains.

In the completer sample there was statistical evidence for somewhat greater improvement on the PDS $[F(1,180)=510.5, p<0.001]$, with a correspondingly larger treatment effect size. There was no statistically significant difference between scores at the end of treatment and at follow-up [t(61) $=-1.67, p>0.05]$, indicating that treatment gains had been well maintained an average of 1 year later. Similar improvement was shown on the BDI $[F(1,181)=300.2, p<0.001]$. There was no statistically significant difference between scores at the end of treatment and at follow-up $[t(63)=-1.54, p>0.05]$, indicating that treatment gains had been well maintained over time.

To test whether treatment gains were more likely at the beginning of the programme, when they could have been reflecting a normal recovery process, a correlation was computed between the extent of improvement and the time elapsed since the bombings before receiving treatment. On average, treatment began 321.49 days after the bombings (S.D. $=170.84$, range $=42-768$ days). The correlations with time elapsed were small and non-significant for both the PDS $[r(204)=-0.06]$ and the BDI $[r(206)=-0.07]$, indicating that treatment was equally effective whether it was delivered early or late in the programme.

\section{DSM-IV PTSD}

Outcome data are presented in Table 2. In the intention-to-treat sample there was a statistically highly significant degree of improvement on the PDS $[F(1,124)=308.1, p<0.001]$. Cohen's $d$ was a very substantial 1.87. Reliable clinical improvement was shown by $76.0 \%$ of patients, and $62.4 \%$ showed clinically significant change. There was similarly a large improvement on the BDI scores $[F(1,124)=167.0$, $p<0.001]$, but with a somewhat lower treatment effect size. Reliable clinical improvement was shown by 
Table 2. Treatment outcome details for DSM-IV post-traumatic stress disorder (PTSD)

\begin{tabular}{|c|c|c|c|c|c|c|}
\hline & $n$ & $\begin{array}{l}\text { Pre-treatment, } \\
\text { mean (s.D.) }\end{array}$ & $\begin{array}{l}\text { Post-treatment, } \\
\text { mean (S.D.) }\end{array}$ & $\begin{array}{l}\text { Effect size } \\
\text { (Cohen's } d \text { ) }\end{array}$ & $\begin{array}{l}\text { Effect size based } \\
\text { on difference score } \\
\text { (s.D. of difference) }\end{array}$ & $\begin{array}{l}\text { Follow-up, } \\
\text { mean }(n, \text { S.D. })\end{array}$ \\
\hline \multicolumn{7}{|c|}{ Intention-to-treat sample } \\
\hline PDS & 125 & $34.1(9.3)$ & $13.6(12.6)$ & 1.87 & $1.57(13.0)$ & - \\
\hline BDI & 125 & $25.1(9.9)$ & $12.2(11.1)$ & 1.23 & $1.16(11.3)$ & - \\
\hline \multicolumn{7}{|c|}{ Completer sample } \\
\hline PDS & 104 & $34.4(9.4)$ & $11.6(12.2)$ & 2.11 & $1.88(12.1)$ & $14.9(37,12.5)$ \\
\hline BDI & 104 & $25.2(10.1)$ & 10.6 (10.6) & 1.41 & 1.41 (10.5) & $11.2(39,10.2)$ \\
\hline
\end{tabular}

PDS, Post-traumatic Diagnostic Scale; BDI, Beck Depression Inventory; S.D., standard deviation.

$71.2 \%$ of patients, and $53.6 \%$ showed clinically significant change.

In the completer sample there was once again evidence for pronounced improvement in DSM-IV PTSD on the PDS $[F(1,103)=367.4, p<0.001]$. Reliable clinical improvement was shown by $86.0 \%$ of patients, and $66.4 \%$ showed clinically significant change. There was no statistically significant difference between scores at the end of treatment and at follow-up $[t(36)=-1.40$, $p>0.05]$, indicating that treatment gains had been well maintained over time. There was similarly pronounced improvement on the BDI scores $[F(1,103)=$ 206.2, $p<0.001]$, but again with a somewhat lower treatment effect size. Reliable clinical improvement was shown by $82.2 \%$ of patients, and $56.1 \%$ showed clinically significant change. Once again there was no statistically significant difference between scores at the end of treatment and at follow-up $[t(38)=-1.00$, $p>0.05]$, indicating that treatment gains had been well maintained.

\section{Discussion}

Although general population samples show a steep reduction in post-traumatic symptoms after disasters and terrorism, researchers detailing the clinical response to those most directly affected have commented repeatedly on the level of persistent unmet mental health needs (Call \& Pfefferbaum, 1999; WTC Medical Working Group, 2008). The TRP was designed specifically to address this problem. However, as no reliable estimates or official figures have been published documenting the numbers directly affected by the London bombings, it is not possible to assess what proportion of these were apprised of the programme and what proportion of those who needed treatment received it.

Previous studies suggest that treatment was rare without prior experience of the mental health system (Stuber et al. 2006) and that only a small minority of individuals initiate contact with treatment services themselves (Rosser et al. 1991). Our data confirmed people's reluctance to refer themselves, and worryingly suggested a failure of standard care pathways. Although all GPs in London were informed individually about the programme on more than one occasion, very few of those identified had been referred in this way.

Did this reflect a failure of the primary care pathway? Perhaps GPs referred their patients for treatment elsewhere? We were in contact with other local departments of clinical psychology in London and consider it very unlikely that patients were referred for NICE-approved treatment from other sources. It could also be argued that GPs would have made referrals given sufficient time. We suggest that there are three reasons why this is unlikely: first, international data generally indicate persistent high levels of unmet need after disasters (Pfefferbaum et al. 2002; WTC Medical Working Group, 2008); second, there is evidence that in the UK recognition of PTSD by GPs tends to be poor (Ehlers et al. 2009); and third, qualitative responses given during the course of our study identified a high level of dissatisfaction with GPs (Brewin et al. 2009). This suggests that without the programme's efforts at outreach there would have been much greater levels of unmet need.

In this study $31 \%$ of those who came to the programme's attention, and $47 \%$ of those who were screened, were judged to need treatment. These figures are consistent with the finding that $30-40 \%$ of those exposed directly to a terrorist attack are likely to suffer from PTSD 2 years later (Whalley \& Brewin, 2007). Nevertheless, the fact that such a high proportion screened positive may have reflected a lack of representativeness in our sample. In addition to variation in the way in which people were referred to the programme, the great majority of individuals had been affected directly, either by being injured themselves or by witnessing others' injuries. Many were 
convinced they would die. All these experiences are established risk factors for developing difficulties such as PTSD.

The outcome data add to evidence from previous, smaller studies that established psychological treatment methods can substantially reduce PTSD (Duffy et al. 2007; Levitt et al. 2007) in those affected by terrorist attacks, and show for the first time that these gains are maintained at an average of 1 year follow-up. Reporting a previous mental health problem did not result in a poorer outcome, and those with travel phobia also responded very strongly to treatment (Handley et al. 2009). The easiest way of evaluating our outcomes against other studies is to restrict the comparison to RCTs of multi-session CBT for individuals with DSM-IV PTSD following adult trauma (excluding military veterans who are likely to have extended trauma and generally have poorer outcomes). Cahill et al. (2009) have tabulated within-group and betweengroup effect sizes from previous trials. Within-group effect sizes tend to be larger and provide the appropriate comparison for the data from this study. The weighted average of within-group effect sizes for intention-to-treat analyses in previous trials is 1.32 , and for completer analyses is 1.73 . Our own effect sizes of 1.87 and 2.11 support the effectiveness of the therapy provided.

It is important to acknowledge that a significant minority of individuals received far more treatment sessions than are generally recommended by NICE for the treatment of PTSD. This was in most cases due to prior trauma or pre-existing psychopathology that required more extensive intervention. It is well established that such factors increase the risk for PTSD following exposure to trauma (Brewin et al. 2000). Thus, post-disaster service planning needs to incorporate the likelihood that a minority of individuals will require a level of intervention that exceeds the usual recommendations for PTSD following a single trauma.

The study had several limitations, some of which reflect the requirement to mount an effective response to the immediate needs of the affected population, despite the absence of any established mechanism to fund additional clinical activity or research within the necessary time-frame. Among these limitations, the absence of a waiting list control group makes it difficult to establish with certainty that the good outcome was due to treatment rather than natural recovery. However, a previous RCT that used an outreach and screening approach in motor vehicle accident survivors showed that TF-CBT was superior to a waitinglist control (Ehlers et al. 2003). We have additionally shown that people treated later in the programme had the same good outcomes as people treated earlier. As the bulk of natural recovery following trauma usually occurs in the first 6-12 months (Helzer et al. 1987; Kessler et al. 1995), it is highly unlikely that spontaneous improvement could account for the observed outcomes. Another limitation stems from the lack of any mechanism in current UK emergency planning to establish a comprehensive database of all those who are affected by a disaster. This may lead to foreign nationals who return home post-incident being overlooked, and necessarily restricts the generalizability of all data, including ours, that seek to assess postdisaster outcomes.

There is now sufficient evidence to anticipate substantial levels of unmet need for mental health care following terrorist incidents, unless additional resources are provided. Although screening post-trauma has not always been useful in a military context (Rona et al. 2004), there have now been several implementations in civilian post-disaster settings such as the 11 September 2001 WTC attacks (Donahue et al. 2006) and Hurricane Katrina (Hamblen et al. 2009). In both of these programmes screening was introduced at least 18 months post-disaster and without being linked to extensive outreach. Our data go further in suggesting that, in the context of a terrorist attack, early and systematic attempts at outreach and screening may be an effective way of identifying those who could benefit from treatment, and that outreach can be coordinated with existing or specially commissioned resources to increase access to effective therapy. Important unanswered questions remain, for example about whether the approach would be effective for disasters where there are high levels of infrastructure damage, or where large numbers of children or survivors lacking English as a first language are involved. More rigorous testing of screening and alternative approaches and more systematic methods of identifying those involved in an incident are now urgently required.

\section{Acknowledgements}

Funding was received from the Department of Health Forensic Mental Health Programme, and research support from Camden and Islington NHS Foundation Trust. Dr Ehlers is supported by the Wellcome Trust.

\section{Declaration of Interest}

None.

\section{References}

Beck AT, Rush AJ, Shaw BF, Emery G (1979). Cognitive Therapy of Depression. Guilford Press: New York.

Brewin CR (2001). Cognitive and emotional reactions to traumatic events: implications for short-term intervention. Advances in Mind-Body Medicine 17, 163-168. 
Brewin CR (2005). Systematic review of screening instruments for the detection of posttraumatic stress disorder in adults. Journal of Traumatic Stress 18, 53-62.

Brewin CR, Andrews B, Valentine JD (2000). Meta-analysis of risk factors for posttraumatic stress disorder in traumaexposed adults. Journal of Consulting and Clinical Psychology 68, 748-766.

Brewin CR, Fuchkan N, Huntley Z (2009). Evaluation of the NHS Trauma Response to the London Bombings (MRD 12/98). Final Report to the Department of Health Forensic Mental Health R\&D Programme. Department of Health: London.

Brewin CR, Rose S, Andrews B, Green J, Tata P, McEvedy C, Turner SW, Foa EB (2002). A brief screening instrument for posttraumatic stress disorder. British Journal of Psychiatry 181, 158-162.

Brewin CR, Scragg P, Robertson M, Thompson M, d'Ardenne P, Ehlers A (2008). Promoting mental health following the London bombings: a screen and treat approach. Journal of Traumatic Stress 21, 3-8.

Cahill SP, Rothbaum BO, Resick PA, Follette VM (2009). Cognitive-behavioral therapy for adults. In Effective Treatments for PTSD (ed. E. B. Foa, T. M. Keane, M. J. Friedman and J. A. Cohen), pp. 139-222. Guilford: New York.

Call JA, Pfefferbaum B (1999). Lessons from the first two years of Project Heartland, Oklahoma's mental health response to the 1995 bombing. Psychiatric Services 50, 953-955.

Donahue S, Jackson C, Shear MK, Felton C, Essock S (2006). Outcomes of enhanced counseling services provided to adults through Project Liberty. Psychiatric Services 57, 1298-1303.

Duffy M, Gillespie K, Clark DM (2007). Post-traumatic stress disorder in the context of terrorism and other civil conflict in Northern Ireland: randomised controlled trial. British Medical Journal 334, 1147-1150.

Ehlers A, Clark DM, Hackmann A, McManus F, Fennell M, Herbert C, Mayou R (2003). A randomised controlled trial of cognitive therapy, a self-help booklet, and repeated assessment as early interventions for PTSD. Archives of General Psychiatry 60, 1024-1032.

Ehlers A, Gene-Cos N, Perrin M (2009). Low recognition of post-traumatic stress disorder in primary care. London Journal of Primary Care 2, 36-42.

First MB, Spitzer RL, Gibbon M, Williams JBW (1997). Structured Clinical Interview for DSM-IV Axis I Disorders Patient Edition (SCID-IP; Version 2.0, 4/97 revision). Biometrics Research Department, New York State Psychiatric Institute: New York.

Foa EB, Cashman L, Jaycox L, Perry K (1997). The validation of a self-report measure of posttraumatic stress disorder: the Posttraumatic Diagnostic Scale. Psychological Assessment 9, 445-451.

Gillespie K, Duffy M, Hackmann A, Clark DM (2002). Community based cognitive therapy in the treatment of posttraumatic stress disorder following the Omagh bomb. Behaviour Research and Therapy 40, 345-357.

Hamblen JL, Norris FH, Pietruszkiewicz S, Gibson LE, Naturale A, Louis C (2009). Cognitive behavioral therapy for postdisaster distress: a community based treatment program for survivors of Hurricane Katrina. Administration and Policy in Mental Health and Mental Health Services Research 36, 206-214.

Handley RV, Salkovskis PM, Ehlers A (2009). Treating clinically significant avoidance of public transport following the London bombings. Behavioural and Cognitive Psychotherapy 37, 87-93.

Helzer JE, Robins LN, McEvoy L (1987). Post-traumatic stress disorder in the general population: findings of the Epidemiological Catchment Area Survey. New England Journal of Medicine 317, 1630-1634.

Jacobson NS, Truax P (1991). Clinical significance: a statistical approach to defining meaningful change in psychotherapy research. Journal of Consulting and Clinical Psychology 59, 12-19.

Kessler RC, Sonnega A, Bromet E, Hughes M, Nelson CB (1995). Posttraumatic stress disorder in the National Comorbidity Survey. Archives of General Psychiatry 52, 1048-1060.

Kroenke K, Spitzer RL, Williams JBW (2003). The Patient Health Questionnaire-2: validity of a two-item depression screener. Medical Care 41, 1284-1292.

Levitt JT, Malta LS, Martin A, Davis L, Cloitre M (2007). The flexible application of a manualised treatment for PTSD symptoms and functional impairment related to the 9/11 World Trade Center attack. Behaviour Research and Therapy 45, 1419-1433.

Neria Y, Nandi A, Galea S (2008). Post-traumatic stress disorder following disasters: a systematic review. Psychological Medicine 38, 467-480.

NICE (2005). Post-traumatic Stress Disorder: the Management of PTSD in Adults and Children in Primary and Secondary Care. National Institute for Clinical Excellence: London.

Pfefferbaum B, North CS, Flynn BW, Norris FH, DeMartino R (2002). Disaster mental health services following the 1995 Oklahoma City bombing: modifying approaches to address terrorism. CNS Spectrums $\mathbf{7}$, 575-579.

Rona RJ, Jones M, French C, Hooper R, Wessely S (2004). Screening for physical and psychological illness in the British Armed Forces. I: The acceptability of the programme. Journal of Medical Screening 11, 148-152.

Rosser R, Dewar S, Thompson J (1991). Psychiatric aspects of disaster: psychological aftermath of the King's Cross fire. Journal of the Royal Society of Medicine 84, 4-8.

Rubin GJ, Brewin CR, Greenberg N, Hacker Hughes J, Simpson J, Wessely S (2007). Enduring consequences of terrorism: 7-month follow-up survey of reactions to the bombings in London on 7 July 2005. British Journal of Psychiatry 190, 350-356.

Rubin GJ, Brewin CR, Greenberg N, Simpson J, Wessely S (2005). Psychological and behavioural reactions to the 7 July London bombings: a cross-sectional survey of a representative sample of Londoners. British Medical Journal 331, 606-611.

Seggar LB, Lambert MJ, Hansen NB (2002). Assessing clinical significance: application to the Beck Depression Inventory. Behavior Therapy 33, 253-269. 
Stuber J, Galea S, Boscarino JA, Schlesinger M (2006). Was there unmet mental health need after the September 11, 2001 terrorist attacks? Social Psychiatry and Psychiatric Epidemiology 41, 230-240.

Turner SW, Thompson JA, Rosser RM (1989). The King's Cross fire: planning a 'phase two' psychosocial response. Disaster Management 2, 31-37.

Walters JTR, Bisson JI, Shepherd JP (2007). Predicting post-traumatic stress disorder: validation of the Trauma
Screening Questionnaire in victims of assault. Psychological Medicine 37, 143-150.

Whalley MG, Brewin CR (2007). Mental health following terrorist attacks. British Journal of Psychiatry 190, 94-96.

WTC Medical Working Group (2008). Annual Report on 9/11 Health. World Trade Center Medical Working Group of New York City: New York. 